\title{
nature
}

biotechnology

\section{Outpaced by an outbreak}

\author{
The international community's woeful response to the West African Ebola epidemic has been compounded by the \\ lack of vaccines and therapies on the ground. That is why US government cutbacks to biodefense funding should be \\ reevaluated and five-year funding tranches restored.
}

The

he human tragedy unfolding in Guinea, Sierra Leone and Liberia due to the spread of Ebola hemorrhagic fever was eminently preventable. The World Health Organization (WHO) had ample time to mobilize the international community-yet failed to do so despite repeated calls from Médecins Sans Frontières doctors battling the contagion since March. National governments also allowed a $50 \%$ budget cut to WHO that forced a downsizing of emergency response units and the layoff of large numbers of epidemic control experts in the field. Until August, the US government (the leading state donor) was devoting a paltry $\$ 2.1$ million per month to the Ebola response; finally, in October, more was allocated- $\$ 1.25$ billion more, plus a commitment of 3,000 military personnel. The threat from Ebola was as clear a danger as any of the traditional contagions, like anthrax or smallpox, just not as present. Out of sight, out of mind. With no approved vaccine or therapeutic against the virus, the worry is that the threat could get out of control.

Previous outbreaks of Ebola have been limited in size and geographic spread, typically affecting a handful to a few hundred people in rural settings in Central Africa. This epidemic is different. It covers a huge expanse of West Africa (at one stage it also included Senegal and Nigeria). It affects poor countries with unstable governments. It is occurring in urban settings rather than in isolated rural areas where it is more easily contained. It is being exacerbated by local burial customs and mistrust of outsiders. Local healthcare systems are simply failing under the appalling burden of disease. As Nature Biotechnology went to press, an unprecedented $~ 10,000$ cases of Ebola had been reported, with 4,877 deaths.

In the past, Ebola has been countered through a combination of early diagnosis, patient isolation, contact tracing, infection control, community engagement and safe burial. This time, the virus is outpacing the capacity of local authorities to maintain infection control. As the disease has spread, health workers and missionaries who have contracted the virus have been offered experimental therapies on a compassionate-use basis (outside of a controlled clinical trial). But there is not enough to go around.

For example, ZMapp, a monoclonal antibody cocktail that gained widespread media attention has been used in only seven patients. The next lot, scheduled for December, will be for only $\sim 20$ more treatment courses. Tekmira Pharmaceuticals will have 54 aliquots of its short interfering RNA drug by the end of the year and 490 by March 2015. Sarepta has up to 24 treatment courses of its antisense therapeutic. BioCryst is planning to file an Investigational New Drug Application this month for its small molecule BCX4430, with 2,000 treatment courses due in March.

The news for vaccine availability is slightly better. GlaxoSmithKline and the US National Institute for Allergy and Infectious Disease have initiated a single-dose, phase 1 trial of a chimpanzee adenovirus 3 vaccine, with 15,000 doses available by the end of the year. NewLink Genetics and the Public Health Agency of Canada's vaccine is being ramped up to production of 1,400 doses. Johnson \& Johnson/Crucell/Bavarian Nordic, Profectus BioSciences and Thomas Jefferson University also are developing Ebola vaccines.

In September, the US Biomedical Advanced Research and Development Authority (BARDA) announced it would be extending a contract of up to \$42.3 million to Mapp Pharmaceuticals to scale up ZMapp production. Tekmira and BioCryst are also receiving funding from the US Department of Defense (DoD).

These grants follow BARDA's establishment two years ago of three US public-private partnerships to create centers with the specific purpose of manufacturing vaccines and medicine on a commercial scale, with an emphasis on a flexible, rapid response in the face of a public health emergency (like Ebola). The first partnership is between Emergent BioSolutions, Michigan State University, Kettering University and the University of Maryland, Baltimore; a second is between Novartis (which recently opened a cell-based, pandemic flu vaccine production facility), North Carolina State University and Duke University; and a final consortium involves The Texas A\&M University System, GlaxoSmithKline and Kalon Biotherapeutics. Nine months ago, BARDA also established a network of five clinical research organizations.

So BARDA has made judicious progress in establishing the infrastructure to respond to an epidemic. The current crisis just arrived a few years too soon.

We have no medicines to combat Ebola partly because of the time it takes to develop products-BARDA has been in existence for only ten years; partly because of red tape and bureaucracy-ZMapp was held up for two years by DoD before it funded the program in 2013; partly because of the difficulty of prioritizing countermeasures and biological threats from behind a desk in Washington, DC; and partly, and most recently, because of the fiduciary handcuffs placed on BARDA.

In 2013, the US Congress changed BARDA's budgeting rules so that instead of providing funding for five years, the agency must now work on the basis of annual appropriations; in FY '14, these were a niggardly \$255 million for Project BioShield and \$415 million for BARDA. The question is, with its budgeting limited to cheeseparing annual handouts, can the agency do the type of long-term planning needed to propel countermeasures for Ebola, or any other agent for the matter, to the market?

The largest Ebola epidemic in history underlines the urgency of creating countermeasures not only for emerging pathogens like pandemic flu but also for known pathogens that behave in new ways in an increasingly interconnected, urbanized and overcrowded world. The fact that outbreaks will occur and they will evolve is entirely predictable. Credible biodefense demands the sustainability that the corporate profit motive can provide. That means that government funding for biodefense must be long term and commensurate with the real level of threat. 